\title{
Chronic $\Delta^{9}$-Tetrahydrocannabinol During Adolescence Increases Sensitivity to Subsequent Cannabinoid Effects in Delayed Nonmatch-to-Position in Rats
}

\author{
Jenny L. Wiley ${ }^{1}$ and James J. Burston ${ }^{1,2}$ \\ ${ }^{1}$ Department of Pharmacology and Toxicology, Virginia Commonwealth University, Richmond, \\ Virginia \\ ${ }^{2}$ Faculty of Applied Sciences, University of the West of England, Frenchay Campus, Coldharbour \\ Lane, Bristol, UK
}

\begin{abstract}
Early-onset marijuana use has been associated with short- and long-term deficits in cognitive processing. In human users, self-selection bias prevents determination of the extent to which these effects result only from drug use. This study examined the long-term effects of $\Delta^{9}$ tetrahydrocannabinol $\left(\Delta^{9}-\mathrm{THC}\right)$, the major psychoactive constituent of marijuana, in a delayed nonmatch-to-position task (DNMP). Male Long-Evans rats were injected daily with $10 \mathrm{mg} / \mathrm{kg} \Delta^{9}$ THC during or after adolescence [postnatal days (PN) 21-50 or PN50-79, respectively] or with vehicle. On PN91, training in DNMP was initiated. Successful acquisition and pharmacological challenge began on approximately PN300. Decreases in accuracy were observed at lower doses of $\Delta^{9}$-THC in $\Delta^{9}$-THC-treated rats (versus vehicle-treated rats). Administration of chronic $\Delta^{9}$-THC at a younger age tended to enhance this effect. While anandamide did not decrease accuracy in any group, rats treated with $\Delta^{9}$-THC during adolescence initiated fewer trials at the $30 \mathrm{mg} / \mathrm{kg}$ dose of anandamide than did rats in the other two groups. To the extent tested, these differences were pharmacologically selective for cannabinoids, as scopolamine (positive control) decreased accuracy at the same dose in all groups and amphetamine (negative control) did not affect accuracy in any of the groups at doses that did not impair overall responding. These results suggest that repeated administration of a modest dose of $\Delta^{9}$-THC during adolescence (PN21-50) or shortly thereafter (PN50-79) produces a long-term increase in latent sensitivity to cannabinoid-induced impairment of performance in a complex operant task.
\end{abstract}

\section{Keywords}

adolescence; amphetamine; anandamide; cannabinoids; delayed nonmatch-to-position; long-term effects; scopolamine; $\Delta^{9}$-tetrahydrocannabinol

\begin{abstract}
Adolescence is characterized by rapid change in many domains, including physical, emotional, social, and cognitive. During this period, substantial re-organization of receptors for several neurotransmitter systems in the brain, including $\mathrm{CB}_{1}$ receptors (Mato et al., 2003), occurs,
\end{abstract}

Please send correspondence to: Jenny Wiley, Ph.D., Dept. of Pharmacology \& Toxicology, Virginia Commonwealth University, Box 980613, Richmond, Virginia 23298-0613, (804) 828-2067 (phone), (804) 828-2117 (FAX), jwiley@ vcu.edu.

Publisher's Disclaimer: This is a PDF file of an unedited manuscript that has been accepted for publication. As a service to our customers we are providing this early version of the manuscript. The manuscript will undergo copyediting, typesetting, and review of the resulting proof before it is published in its final citable form. Please note that during the production process errors may be discovered which could affect the content, and all legal disclaimers that apply to the journal pertain. 
allowing for the rapid development of advanced cognitive skills that are required for academic achievement and for transition into adult roles. In addition, increased independence during this period is often accompanied by experimentation with novel ideas and behaviors that may include recreational drug use. Some of these drugs, including marijuana (the most frequently used illicit substance during adolescence), have been shown to produce cognitive deficits in adult users [for review, see (Ranganathan and D'Souza, 2006)]. Review of the few studies that have investigated the effects of marijuana during adolescence reveals that adolescent marijuana users exhibit both short- and long-term deficits in cognitive processing, even after various periods of abstinence. For example, marijuana use during adolescence was associated with abnormal findings in patterns of brain activation during cognitive tasks suggestive of altered processing of information and increased effort during tasks requiring inhibition of a response (Schweinsburg et al., 2008; Tapert et al., 2007). Early marijuana use was also associated with long-term effects, including impaired reaction times in a visual attention task (Ehrenreich et al., 1999), lower levels of academic achievement (Brook et al., 2008), and continued reductions in brain activation in prefrontal and cerebellar regions (Chang et al., 2006). Determination of mechanisms that may underlie these marijuana-induced deficits, however, is complicated by the fact that human users are self-selected and may differ on important characteristics before initiation of use as well as life trajectory after initiation. Hence, the majority of mechanistic research in this area has been conducted in immature animals.

In rats, adolescence lasts approximately two weeks, from postnatal day (PN) 28 - PN42 (Spear, 2000). This developmental period occurs around the time of puberty and is associated with rapid physical maturation as well as with behavioral changes reflecting increased risk taking and orientation towards conspecifics (Spear, 2000). In addition, dramatic changes in brain cannabinoid $\left(\mathrm{CB}_{1}\right)$ receptors have been reported. $\mathrm{CB}_{1}$ receptors in the rat brain exhibit a progressive increase in number, but not in affinity, during the pre-weanling period (i.e., before PN21) and early adolescence (female peak at PN30 and male peak at PN40), with receptor pruning and a decline to adult levels during later adolescence (Belue et al., 1995; McLaughlin et al., 1994; Rodriguez de Fonseca et al., 1993). By PN60, adult levels of cannabinoid receptors are achieved (Belue et al., 1995). The functional consequences of these changes in brain $\mathrm{CB}_{1}$ receptor composition are most likely to occur in behavioral arenas (e.g., cognition, brain reward, movement, and appetite regulation) that are associated with brain areas with high endocannabinoid involvement. The long-term effects on performance in a memory task after adolescent exposure to $\Delta^{9}$-tetrahydrocannabinol $\left(\Delta^{9}\right.$-THC), the primary psychoactive substituent of the marijuana plant, are the focus of this investigation.

Delayed nonmatch-to-position (DNMP) is an operant procedure that is commonly used to examine the effects of drugs on short-term memory in rats (Dudchenko, 2004). Acquisition of this procedure involves a series of steps, including chamber habituation, lever press training, acquiring rules for choice of correct lever, and memory retention over a span of delay durations. The final procedure consists of discrete trials that are comprised of a sample phase, a delay and a choice phase. During the sample phase, the rat is presented with one of two levers. Both levers are then withdrawn for a varying period of delay. During the subsequent choice phase, both levers are re-inserted into the chamber and the rat must press the opposite lever from that presented in the sample phase in order to receive food reward. In the absence of motor, attentional, or motivational influences, delay-dependent decreases in accuracy of choosing the correct (nonmatch) lever reflect impairment of short-term memory (Dunnett, 1989). In this procedure, $\Delta^{9}$-THC dose-dependently induces delay-dependent accuracy impairments in adult rodents, suggesting interference with one or more processes involved in encoding and/or retrieval of memory (Hampson and Deadwyler, 2000; Heyser et al., 1993). The purpose of the present study was to examine performance in the DNMP procedure in adult rats that previously had been treated chronically with $\Delta^{9}$-THC during and shortly after adolescence. Performance was compared with that of rats chronically treated with vehicle during adolescence. After 
acquisition of the procedure, rats were challenged sequentially with $\Delta^{9}$-THC and anandamide (an endogenous cannabinoid receptor ligand) in order to determine differential inter-group sensitivity to the disruptive effects of these cannabinoids on accuracy in the procedure. For comparison purposes, we also tested scopolamine and amphetamine. Scopolamine is an antagonist at muscarinic acetylcholine receptors and reliably produces impairments in accuracy in this type of procedure (Stanhope et al., 1995). Amphetamine is an indirect agonist of monoamine neurotransmission, including dopamine. Since brain dopamine receptors undergo re-organization during adolescence in parallel with brain $\mathrm{CB}_{1}$ receptors (Tarazi and Baldessarini, 2000), tests with amphetamine served as an initial gauge of whether any differential sensitivity was selective for cannabinoids or generalized to a drug acting at another system undergoing alteration during the same time period.

\section{METHODS}

\section{Animals}

Timed pregnant adult female Long-Evans rats (Harlan, Dublin, VA), ordered for arrival on gestation day 14, were individually housed in clear plastic cages $(52 \times 28 \times 22 \mathrm{~cm})$ with sawdust bedding available in each cage for nesting. The vivarium had a temperature-controlled (20$22^{\circ} \mathrm{C}$ ) environment with a 12 -hour light-dark cycle (lights on at 7 a.m.). The dams were left undisturbed except for providing food, water, and fresh bedding until they gave birth (postnatal day 0 , PNO). Pups were sexed and culled to no more than 10 pups per litter and remained with their dams until weaning at PN21. On PN21, male pups were separated from the dam. Each pup was assigned to one of three treatment groups (described in the procedures section) such that each group contained no more than one pup from a litter. Each rat was pair-housed with a rat from another litter until PN90. All rats had free access to food until they started training in the DNMP procedure on PN91. Subsequently, they received a daily ration of standard rat chow sufficient to maintain their body weight at no less than $85 \%$ of free-feeding weights (as indicated by rat growth chart from Harlan). Throughout the study, water was freely available in home cages. These studies were carried out in accordance with guidelines published in the Guide for the Care and Use of Laboratory Animals (National Research Council, 1996) and were approved by the Institutional Animal Care and Use Committee at Virginia Commonwealth University.

\section{Apparatus}

Rats in each procedure were trained and tested in standard operant conditioning chambers (BRS/LVE Inc., Laurel, MD and Lafayette Instruments Co., Lafayette, IN) housed in soundattenuated cubicles. Pellet dispensers delivered 45-mg BIO SERV (Frenchtown, NJ) food pellets to a food cup on the front wall of the chamber between two retractable response levers. Fan motors provided ventilation and masking noise for each chamber. House lights located above each retractable lever were illuminated during training and testing sessions. A microcomputer with Logic ' 1 ' interface (MED Associates, Georgia, Vermont) and MED-PC software (MED Associates) was used for these tasks.

\section{Drugs}

$\Delta^{9}$-THC [National Institute on Drug Abuse (NIDA), Bethesda, MD] and anandamide (NIDA) were mixed in a vehicle of absolute ethanol, Emulphor-620 (Rhone-Poulenc, Inc., Princeton, $\mathrm{NJ}$ ), and saline in a ratio of 1:1:18. Amphetamine sulfate (NIDA) and (-)-scopolamine $\mathrm{HBr}$ (Sigma-Aldrich, St. Louis, MO) were mixed in sterile water. Injections of all test drugs were administered intraperitoneally at a volume of $1 \mathrm{ml} / \mathrm{kg}$. Pre-session injection intervals were 30 $\min$ for $\Delta^{9}$-THC and scopolamine, $10 \mathrm{~min}$ for anandamide and $15 \mathrm{~min}$ for amphetamine. Drugs were tested in the following order: $\Delta^{9}$-THC, scopolamine, anandamide, and amphetamine. Doses of each drug were presented according to a randomized Latin square design. 


\section{Procedure}

Rats were assigned to one of three treatment conditions. Rats in the first and second groups were injected s.c. with $10 \mathrm{mg} / \mathrm{kg} \Delta^{9}$-THC or vehicle, respectively, once daily from PN21-50. Rats in the third group were injected s.c. with $10 \mathrm{mg} / \mathrm{kg} \Delta^{9}$-THC once daily from PN50-79. Subsequently, rats in all three groups were allowed to remain in their cages undisturbed except for weighing at least twice a week until PN90. Hence, rats in each group differed in age at $\Delta^{9}$-THC exposure, but were approximately the same age during DNMP training and testing.

On PN91, acquisition training for the DNMP procedure was initiated for all groups using a regimen similar to the behavioral shaping procedure that we have used previously (Wiley and Willmore, 2000). First, magazine training was undertaken, consisting of habituation to the chambers (without levers) and noncontingent pellet delivery every $30 \mathrm{~s}$ for $20 \mathrm{~min}$. Once rats were reliably eating the pellets autoshaping of the lever press began. During this phase of training, the left and right levers were inserted into the chamber on alternate days. The number of times the rat was required to press each lever was increased gradually from 1 to 5 until rats reliably pressed either lever when it was inserted into the chamber. A trials procedure was then instigated with alternate levers inserted into the chamber during a single session, with 5 lever presses required on the presented lever for pellet delivery. A $10 \mathrm{~s}$ inter-trial interval occurred, during which levers were withdrawn and all lights were turned off. Subsequently, a choice procedure was implemented and was maintained through acquisition and testing. Under the choice procedure, the session length was $30 \mathrm{~min}$. Each trial in the session consisted of three phases: the sample phase, the delay phase, and the choice phase. A trial began with the presentation of either the left or the right response lever (the sample stimulus) accompanied by illumination of the corresponding lever light. The order of sampled levers was random and counterbalanced. Subjects continued each trial by making an observing response [5 lever presses (FR5)] at the extended lever, which produced a food reward. After the observing response, the lever retracted and a delay phase began, which varied in length from 1 to $60 \mathrm{~s}$. Thirteen delay intervals were programmed for possible presentation: $1,5,10,15, \ldots 55,60 \mathrm{~s}$. A random order of presentation was arranged for the delays, and all were equiprobable. Lever lights above the left and right response levers were programmed to blink on and off rapidly during delay intervals. In order to discourage the use of positional strategies (e.g., standing near the correct lever) to choose the correct lever, a noncontingent pellet was delivered every $10 \mathrm{~s}$ during delay periods longer than $10 \mathrm{~s}$. At the end of the scheduled delay, the lights stopped flashing, but remained lit. This marked a transition into choice phase, with both response levers reinserting into the chamber. A press at the lever that did not match the sampled lever (i.e., the alternate lever to that which was previously presented) resulted in retraction of both levers and food pellet delivery. A press of the incorrect lever (i.e., the same lever that was presented in the sample phase of the trial) resulted in lever retraction only. If neither lever was pressed within $15 \mathrm{~s}$ after insertion into the chamber, both levers were retracted (i.e., limited hold of $15 \mathrm{~s}$ ) and the trial was counted as an omission (of choice behavior). After levers were retracted, a $5 \mathrm{~s}$ time out period occurred before the start of the next trial. During time out periods, both sample levers were retracted and lever lights were extinguished. Trial number for each session varied because trial to trial progression depended upon subject response patterns. Training continued on this schedule until rats obtained $70 \%$ overall accuracy for at least 5 days. After acquisition, drug tests occurred approximately twice per week, with a minimum of 48 hours elapsing between drug administrations. Daily training sessions continued on weekdays in between tests. Criteria for receiving a drug test were (i) correct (i.e., nonmatched) lever choice responses for $70 \%$ of trials in the most recent training session and (ii) number of trials completed in the most recent training session must equal or exceed 30. 


\section{Data Analysis}

Due to the large number of individual delays $(\mathrm{n}=13)$, delays were grouped into seven intervals $(1 \mathrm{~s}, 5-10 \mathrm{~s}, 15-20 \mathrm{~s}, 25-30 \mathrm{~s}, 35-40 \mathrm{~s}, 45-50 \mathrm{~s}, 55-60 \mathrm{~s})$ for analysis. With the exception of the 1-s delay interval, all intervals were of the same duration. The 1-s delay interval was shown separately in order to provide a way to gauge the effects of the drug on choice behavior with minimal delay between sample and choice. Any decreases in accuracy for this interval are likely to be related to deficits in overall ability to perform the task rather than to memory or other cognitive skills. Throughout the study, a within-subject design was used such that each rat received each dose of a drug. Means ( $\forall$ SEM) for accuracy (correct trials expressed as a percentage of total trials initiated) were calculated for each drug dose at each delay interval. In addition, means ( $\forall$ SEM) for the total number of trials and for the total number of trials omitted were calculated for each drug dose.

In the cases where total trial number was decreased, an assumption was made that performance factors (e.g., sensory or motor deficits) were likely to interfere with responding; hence, accuracy values for an individual rat were eliminated from analysis when the rat initiated fewer than 20 trials during the session. This practice can complicate statistical analysis and interpretation of data from repeated measures designs because of the requirement for data at each dose for every subject, with absence of these data at a dose typically resulting in elimination of data for the subject at all doses. Since more than half of the rats in each group did not meet the minimum trial criterion ( $>20$ trials for the entire session) when administered the highest dose of each drug (except anandamide), accuracy data for these doses were not included in analysis. In order to include data for lower doses when more than half of the rats met the minimum trial criterion, mean accuracy for these rats was substituted for each rat that did not meet the criterion. Data for all rats at all doses were included in analysis of drug effects on total numbers of trials and omissions.

Separate mixed design factorial ANOVAs were used to analyze data on total number of trials and omissions for each drug, with group and dose as between- and within-subject factors, respectively. Data on accuracy for each drug were analyzed with two-factor repeated measures ANOVAs (dose $X$ delay interval) for each group. Tukey post hoc tests $(\alpha=0.05)$ were used to specify differences revealed by significant ANOVAs. For each drug, ANOVA and post hoc testing was used to determine which doses affected performance for the group compared to its own vehicle control.

\section{RESULTS}

Rats in all three treatment groups were trained during the same time span and were exposed to identical training regimens. Acquisition of the initial steps of the DNMP task (e.g., magazine and lever press training) began on PN91. Although systematic differences were not observed in these initial steps, vehicle-treated rats required more sessions to reach criteria for the choice component of the final procedure (> 70\% accuracy for at least 5 days) than did rats of either age that were treated with $\Delta^{9}$-THC. Pharmacological challenges were initiated when rats were approximately PN300. Despite the additional sessions required for acquisition in the vehicletreated rats, differences in percent accuracy and the number of correct choices across delay intervals did not significantly differ across the three groups following vehicle administration (i.e., group main effects and group $\mathrm{X}$ delay interactions were not significant for any of the ANOVAs on data for vehicle doses of each drug) [data not shown]. Other measures that were similar across groups included the number of trials in each delay interval (4-5 trials for each of the 13 intervals), the number of food pellets received, the number of trials for the session, and the distribution of responses on the left and right levers (data not shown). In addition, the lever associated with correct choices did not systematically vary across doses of any of the drugs (i.e., no lever bias) [data not shown]. 
Figure 1 shows the effects of $\Delta^{9}$-THC on accuracy across delay intervals in the DNMP task in rats treated chronically with vehicle (left panels) or $\Delta^{9}$-THC during adolescence (PN21-50; middle panels) or with $\Delta^{9}$-THC after adolescence (PN50-79; right panels). In rats treated chronically with vehicle during adolescence, doses of $\Delta^{9}$-THC up to $10 \mathrm{mg} / \mathrm{kg}$ did not significantly affect accuracy [Fig. 1, top left panel; main effect of dose: $F(5,45)=2.7, p<0.05$ ]. (For all figures, dose main effects are indicated by filled symbols whereas symbols for effects of doses that do not differ from vehicle are unfilled.) In contrast, chronic treatment with $\Delta^{9}$ THC during or after adolescence significantly decreased accuracy at lower $\Delta^{9}$-THC doses of $3 \mathrm{mg} / \mathrm{kg}$ (adolescents) and $10 \mathrm{mg} / \mathrm{kg}$ (both groups) [Fig. 1, top middle and right panels; main effects of dose: $F(5,50)=6.1, p<0.05$ and $F(5,45)=3.0$, $p<0.05$ for adolescent and postadolescent dosing, respectively]. Administration of $30 \mathrm{mg} / \mathrm{kg} \Delta^{9}$-THC eliminated responding in most of the rats and was not included in the accuracy analysis and is not shown on the graphs. In order to improve visibility of significant results, accuracy data for $\Delta^{9}$-THC doses of $0.1,0.3$ and $1 \mathrm{mg} / \mathrm{kg}$ also are not presented on the graphs, although they were included in the analyses. Reductions in accuracy for all groups occurred primarily at shorter delays and were not sustained across longer delay intervals, a finding that may have been related in part to delaydependent decreases in baseline (vehicle) accuracy at longer delays. In all three groups, 10 and $30 \mathrm{mg} / \mathrm{kg} \Delta^{9}$-THC significantly decreased total number of trials as compared to vehicle [Fig. 1, bottom panels; main effect of dose, ANOVA on trial number data: $F(6,124)=47.5, p<0.05]$. The $10 \mathrm{mg} / \mathrm{kg}$ dose also significantly increased omissions [dose main effect: $\mathrm{F}(6,167)=6.0$, $\mathrm{p}<0.05]$, although the magnitude of the effect was small.

Figure 2 shows the effects of anandamide on accuracy and on the number of completed trials and omissions. At doses up to $30 \mathrm{mg} / \mathrm{kg}$, anandamide did not affect accuracy in rats treated with chronic vehicle during adolescence (top left panel) or in those treated with chronic $\Delta^{9}$ THC during or after adolescence (top middle and right panels, respectively). In rats treated with chronic $\Delta^{9}$-THC during adolescence, the $30 \mathrm{mg} / \mathrm{kg}$ dose of anandamide significantly reduced the number of trials completed [Fig. 2, bottom middle panel; dose $\mathrm{X}$ group interaction: $F(8,112)=3.1, p<0.05]$. Although statistically significant, the decrease in number of total trials was small in magnitude. Anandamide did not affect the number of omissions in any group (bottom panels).

Figure 3 shows the effects of scopolamine on responding in the DNMP task. Analysis of accuracy data revealed that $0.03 \mathrm{mg} / \mathrm{kg}$ scopolamine significantly decreased accuracy in all three groups whereas $0.01 \mathrm{mg} / \mathrm{kg}$ did not [Fig. 3, top panels; main effects of dose: $\mathrm{F}(2,16)=6.9$, $\mathrm{p}<0.05$ for vehicle-treated rats; $\mathrm{F}(2,18)=25.0, \mathrm{p}<0.05$ for rats treated with $\Delta^{9}$-THC during adolescence; $\mathrm{F}(2,18)=7.6, \mathrm{p}<0.05$ for rats treated with $\Delta^{9}$-THC after adolescence]. Few rats in any of the groups met the inclusion criterion for the 0.1 and $0.3 \mathrm{mg} / \mathrm{kg}$ doses of scopolamine; hence, data for these doses were not included in analysis and are not shown on the graph. Significant reductions in number of trials occurred after injection with all doses of scopolamine $(0.01-0.3 \mathrm{mg} / \mathrm{kg})$ across all treatment groups [Fig. 3, bottom panels; main effect of dose: $\mathrm{F}$ $(4,112)=68.7, p<0.05]$. Dose-dependent increases in omissions were seen at doses of $0.03,0.1$ and $0.3 \mathrm{mg} / \mathrm{kg}$ scopolamine [Fig. 3, bottom panels; dose main effect: $F(4,111)=21.0, \mathrm{p}<0.05$ ].

The pattern of effects produced by amphetamine in the DNMP task was similar across the three treatment groups (Fig. 4). Neither $0.1 \mathrm{mg} / \mathrm{kg}$ nor $0.3 \mathrm{mg} / \mathrm{kg}$ amphetamine affected accuracy in any of the groups as compared to respective vehicle conditions (Fig. 4, top panels). Higher doses of 1 and $3 \mathrm{mg} / \mathrm{kg}$ amphetamine resulted in few or no rats responding in the task. Consequently, accuracy data for these doses were not included in the analysis. Not surprisingly, these doses were associated with significant decreases in the number of trials [Fig. 4, bottom panels; main effect of dose for trial number: $F(4,100)=112.5$, $\mathrm{p}<0.05]$. Omissions were not affected by any dose. 


\section{DISCUSSION}

The long-term effects of cannabinoids on cognitive tasks in adolescent rats are not wellcharacterized. In the present study, adolescent rats were treated chronically with $\Delta^{9}$-THC or vehicle and then trained in a DNMP task, an operant procedure that involves a multi-step training process. Initiation of the first step of DNMP training was undertaken when rats in all treatment groups were young adults (PN91) and pharmacological challenges did not begin until approximately PN300; hence, acquisition training was not contiguous with chronic $\Delta^{9}$-THC or vehicle treatment. When injected with vehicle at the start of each dose-effect curve determination, rats in each group showed similar baseline (vehicle) percent accuracies (range $57-95 \%$ across delay intervals, excluding 1-s delay), with pronounced delay-dependent decreases in accuracy at longer intervals.

Consistent with the results of the present study, Stiglick and Kalant (Stiglick and Kalant, 1983) reported that male rats treated with either vehicle or $\Delta^{9}$-THC during adolescence attained the same degree of accuracy in a radial arm maze task during a subsequent drug-free period, despite initial delays in acquisition of the task by $\Delta^{9}$-THC-treated rats. The few additional studies that have assessed the long-term effects of prior chronic cannabinoid administration during adolescence on later performance in cognitive tasks have produced conflicting results, albeit differences in experimental parameters (e.g., rat strain, choice of cannabinoid agonist, dosing regimen) somewhat complicate direct comparisons. For example, chronic treatment of male and female adolescent rats with $\Delta^{9}$-THC for 21 days failed to alter their subsequent acquisition of a spatial learning task after a four-week drug-free period (Cha et al., 2007; Cha et al., 2006). In contrast, chronic treatment with oral $\Delta^{9}$-THC or cannabis extract for 3-6 months impaired subsequent learning in a radial arm maze in male rats if treatment commenced during adolescence, but not if it started later (Stiglick and Kalant, 1982; Stiglick and Kalant, 1983; Stiglick and Kalant, 1985). Similarly, chronic periadolescent treatment with the synthetic cannabinoids, WIN 55,212-2 or CP 55,940, or with $\Delta^{9}$-THC decreased drug-free performance in an object recognition task that was conducted after the rats reached maturity (O'Shea et al., 2004; Quinn et al., 2008; Schneider and Koch, 2002).

Although cannabinoid treatment did not result in similar differences in drug-free performance here, the design of the present experiment differs from previous studies described above in at least two fundamental ways. First, the tasks used in the previous studies were relatively noncomplex and did not require an extensive acquisition period. The extended duration of training in the present study may have contributed to the lack of inter-group differences in the vehicle conditions for each group because it allowed all groups to reach a common level of accuracy in the procedure before testing. In addition, it was associated with a drug-free interval that exceeded any of those used in other studies. A second major methodological distinction is examination of the effects of pharmacological challenge on performance in the task. In the present study, rats remained drug-free during the period after chronic $\Delta^{9}$-THC or vehicle administration and throughout acquisition; however, they were subsequently tested in doseeffect determinations for four test drugs (i.e., $\Delta^{9}$-THC, anandamide, scopolamine and amphetamine). In contrast, rats in the previously cited studies did not receive further pharmacological treatment following acquisition of the task.

Latent differences among groups that are not apparent from observation of similar baseline performances may be revealed through pharmacological challenge, as we have observed previously in a spatial alternation task following early perinatal treatment with phencyclidine (Wiley et al., 2003). In the present study, a history of $\Delta^{9}$-THC administration was associated with differential sensitivity to the effects of $\Delta^{9}$-THC, but not anandamide, on accuracy in DNMP performance. Whereas doses of $\Delta^{9}$-THC up to $30 \mathrm{mg} / \mathrm{kg}$ did not impair accuracy in the vehicle-treated rats, both groups that received daily administration of $\Delta^{9}$-THC exhibited 
decreases in accuracy at $10 \mathrm{mg} / \mathrm{kg}$, with $3 \mathrm{mg} / \mathrm{kg}$ decreasing accuracy only in rats treated during adolescence. This latter group was also more sensitive to the effects of anandamide on trial number, although anandamide-induced decreases in accuracy were not seen with any of the groups. The most likely explanation of anandamide's lack of effect on accuracy is its rapid metabolism via fatty acid amide hydrolase (FAAH; Cravatt et al., 1996). This ideas receives support from a previous report that anandamide decreased performance in spatial memory tasks in FAAH $(-/-)$, but not FAAH $(+/+)$, mice (Varvel et al., 2006). Together, these results suggest that $\Delta^{9}$-THC administration that occurs subsequent to chronic treatment with $\Delta^{9}$-THC may reveal latent differences in sensitivity to cannabinoid effects on performance in a learned task acquired after the chronic treatment. Further, the finding that lower doses of $\Delta^{9}$-THC and anandamide were required to decrease accuracy or trial number, respectively, in rats treated with $\Delta^{9}$-THC during adolescence suggests that exposure during this developmental phase may result in particular vulnerability to these effects. Important caveats accompany this latter conclusion, as the range of ages at which exposure occurred was not large compared to the lifespan of the rat. Further, the definition of rat adolescence (i.e., PN28-42) is not absolute, but rather, is an approximate time span characterized by a pattern of behaviors and physiological changes (Spear, 2004). Hence, an identical regimen of $\Delta^{9}$-THC administration during later adulthood may have failed to alter subsequent sensitivity to cannabinoid effects in this task. This hypothesis receives limited support from the finding that the older rats in the current study were less sensitive to the effects of one of the cannabinoids (anandamide) on performance in the DNMP task. On the other hand, Miyamoto et al. (Miyamoto et al., 1995) reported that adult male rats showed increased sensitivity to the effects of $\Delta^{9}$-THC in a delayed match to sample task several days after recovery from repeated administration of $\Delta^{9}$-THC, suggesting that shortterm sensitization to $\Delta^{9}$-THC occurs in adult rats.

Pharmacological selectivity of the between-group differences in sensitivity to performance decrements in the DNMP task was not investigated extensively, as the only noncannabinoids tested were scopolamine and amphetamine. Nevertheless, the pattern of intergroup differences produced by each of these drugs was distinct from that produced by cannabinoids. Similar to $\Delta^{9}$-THC, scopolamine dose-dependently decreased accuracy; however, it did so at the same doses in all groups of rats. In contrast, doses of amphetamine that did not seriously compromise the ability of most rats to perform the task did not affect accuracy in any of the groups. These results suggest that the long-term increase in sensitivity to drug-induced impairment of performance following chronic $\Delta^{9}$-THC treatment shows at least a minimal degree of pharmacological selectivity for cannabinoids.

By contrast with the results presented here for the vehicle-treated rats, most previous studies have found that $\Delta^{9}$-THC and other psychoactive cannabinoids disrupt short-term memory in a variety of spatial tasks, including DNMP, and that they do so in a dose- and delay-dependent manner (Hampson and Deadwyler, 2000; Heyser et al., 1993). Explanation of the discrepancy between the results of this study and the previous studies is difficult without additional research because of the many differences in experimental parameters, including procedural variables (e.g., different tasks), rat strain, magnitude of baseline accuracies, length of training, type of reinforcer, and prior treatment with $\Delta^{9}$-THC or vehicle. Nevertheless, a few points are worth mentioning. First, the lack of $\Delta^{9}$-THC effect in vehicle-treated rats was not due to insufficient dose, as $\Delta^{9}$-THC was tested up to doses that eliminated responding in most rats. Second, although none of the doses of $\Delta^{9}$-THC tested in the vehicle-treated rats produced significant decreases in accuracy compared to vehicle, a trend for decreased accuracy was observed. Finally, the results obtained with scopolamine demonstrate that a prototypic memory-impairing drug produced detrimental effects on the performance of these rats.

The degree to which $\Delta^{9}$-THC-induced decreases in accuracy seen in rats treated with $\Delta^{9}$-THC, but not in those treated with vehicle, represent short-term memory deficits is not clear. 
Certainly, its effects resemble those of scopolamine, the positive control. In addition to its disruption of short-term memory, however, $\Delta^{9}$-THC has also been shown to interfere with attention and timing of responses (Han and Robinson, 2001; Presburger and Robinson, 1999; Verrico et al., 2004; Wiley et al., 2000), suggesting that cannabinoid-induced disruption of one of these cognitive processes may have contributed to the deficits observed here. Interestingly, early cannabis exposure in humans has been associated with impaired performance in a visual attention task (Ehrenreich et al., 1999). Alternatively, the significant decreases in the number of trials completed at the doses affecting accuracy suggests that the nonspecific effects of $\Delta^{9}$ THC on performance (e.g., sedation, motor effects) could also have contributed.

In conclusion, the exact nature of the deficits produced by $\Delta^{9}$-THC in the present study is not entirely certain; however, their differential pattern of occurrence across groups clearly indicates that sensitivity to these deficits was affected by prior exposure to $\Delta^{9}$-THC during adolescence or shortly thereafter. Further, this increased sensitivity occurred following an extended drugfree period before training in the DNMP procedure. Although the majority of persons that try marijuana during adolescence do not go on to become habitual users during adulthood, adolescent onset of regular substance use has been associated with rapid transition to a substance use disorder (Swift et al., 2008; Wittchen et al., 2008). The present results suggest that regular administration of $\Delta^{9}$-THC during adolescence may also have long-term effects on performance in cognitive tasks that remain latent until revealed by later $\Delta^{9}$-THC challenge.

\section{Acknowledgments}

Research supported by National Institute on Drug Abuse grant DA-016644.

\section{References}

Belue R, Howlett A, Westlake T, Hutchings D. The ontogeny of cannabinoid receptors in the brain of postnatal and aging rats. Neurotoxicol Terat 1995;17:25-30.

Brook JS, Stimmel MA, Zhang C, Brook DW. The association between earlier marijuana use and subsequent academic achievement and health problems: A longitudinal study. Am J Addict 2008;17:155-60. [PubMed: 18393060]

Cha YM, Jones KH, Kuhn CM, Wilson WA, Swartzwelder HS. Sex differences in the effects of delta9tetrahydrocannabinol on spatial learning in adolescent and adult rats. Behav Pharmacol 2007;18:5639. [PubMed: 17762524]

Cha YM, White AM, Kuhn CM, Wilson WA, Swartzwelder HS. Differential effects of delta9-thc on learning in adolescent and adult rats. Pharmacol Biochem Behav 2006;83:448-55. [PubMed: 16631921]

Chang L, Yakupov R, Cloak C, Ernst T. Marijuana use is associated with a reorganized visual-attention network and cerebellar hypoactivation. Brain 2006;129:1096-112. [PubMed: 16585053]

Cravatt BF, Giang DK, Mayfield SP, Boger DL, Lerner RA, Gilula NB. Molecular characterization of an enzyme that degrades neuromodulatory fatty-acid amides. Nature 1996;384:83-7. [PubMed: 8900284]

Dudchenko PA. An overview of the tasks used to test working memory. Neurosci Biobehav Rev 2004;28:699-709. [PubMed: 15555679]

Dunnett, SB. Comparison of short-term memory deficits in animal models of ageing using an operant delayed response task in rats. In: Squire, LR.; Lindenlaub, E., editors. The biology of memory. New York: F.K. Schattauer-Verlag; 1989. p. 581-603.

Ehrenreich H, Rinn T, Kunert HJ, Moeller MR, Poser W, Schilling L, Gigerenzer G, Hoehe MR. Specific attentional dysfunction in adults following early start of cannabis use. Psychopharmacology (Berl) 1999;142:295-301. [PubMed: 10208322]

Hampson RE, Deadwyler SA. Cannabinoids reveal the necessity of hippocampal neural encoding for short-term memory in rats. Journal of Neuroscience 2000;20:8932-42. [PubMed: 11102504] 
Han CJ, Robinson JK. Cannabinoid modulation of time estimation in the rat. Behav Neurosci 2001;115:243-6. [PubMed: 11256448]

Heyser CJ, Hampson RE, Deadwyler SA. Effects of $\Delta^{9}$-tetrahydrocannabinol on delayed match to sample performance in rats: Alterations in short-term memory associated with changes in task specific firing of hippocampal cells. J Pharmacol Exp Ther 1993;264:294-307. [PubMed: 8380864]

Mato S, Del Olmo E, Pazos A. Ontogenetic development of cannabinoid receptor expression and signal transduction functionality in the human brain. Eur J Neurosci 2003;17:1747-54. [PubMed: 12752773]

McLaughlin CR, Martin BR, Compton DR, Abood ME. Cannabinoid receptors in developing rats: Detection of mrna and receptor binding. Drug Alcohol Depend 1994;36:27-31. [PubMed: 7988356]

Miyamoto A, Yamamoto T, Watanabe S. Effect of repeated administration of d9-tetrahydrocannabinol on delayed matching-to-sample performance in rats. Neuroscience Letters 1995;201:139-42. [PubMed: 8848237]

National Research Council. Guide for the care and use of laboratory animals. Washington, D.C: National Academy Press; 1996.

O'Shea M, Singh ME, McGregor IS, Mallet PE. Chronic cannabinoid exposure produces lasting memory impairment and increased anxiety in adolescent but not adult rats. J Psychopharmacol 2004;18:5028. [PubMed: 15582916]

Presburger G, Robinson JK. Spatial signal detection in rats is differentially disrupted by delta-9tetrahydrocannabinol, scopolamine, and mk-801. Behav Brain Res 1999;99:27-34. [PubMed: 10512569]

Quinn HR, Matsumoto I, Callaghan PD, Long LE, Arnold JC, Gunasekaran N, Thompson MR, Dawson B, Mallet PE, Kashem MA, Matsuda-Matsumoto H, Iwazaki T, McGregor IS. Adolescent rats find repeated delta(9)-thc less aversive than adult rats but display greater residual cognitive deficits and changes in hippocampal protein expression following exposure. Neuropsychopharmacology 2008;33:1113-26. [PubMed: 17581536]

Ranganathan M, D'Souza DC. The acute effects of cannabinoids on memory in humans: A review. Psychopharmacology (Berl) 2006;188:425-44. [PubMed: 17019571]

Rodriguez de Fonseca F, Ramos JA, Bonnin A, Fernandez RJJ. Presence of cannabinoid binding sites in the brain from early postnatal ages. Neuroreport 1993;4:135-8. [PubMed: 8453049]

Schneider M, Koch M. The cannabinoid agonist win 55,212-2 reduces sensorimotor gating and recognition memory in rats. Behav Pharmacol 2002;13:29-37. [PubMed: 11990717]

Schweinsburg AD, Nagel BJ, Schweinsburg BC, Park A, Theilmann RJ, Tapert SF. Abstinent adolescent marijuana users show altered fmri response during spatial working memory. Psychiatry Res 2008;163:40-51. [PubMed: 18356027]

Spear LP. The adolescent brain and age-related behavioral manifestations. Neurosci Biobehav Rev 2000;24:417-63. [PubMed: 10817843]

Spear LP. Adolescent brain development and animal models. Ann N Y Acad Sci 2004;1021:23-6. [PubMed: 15251870]

Stanhope KJ, McLenachan AP, Dourish CT. Dissociation between cognitive and motor/motivational deficits in the delayed nonmatching to position test: Effects of scopolamine, 8-OH-DPAT and EAA antagonists. Psychopharmacology (Berl) 1995;122:268-80. [PubMed: 8748396]

Stiglick A, Kalant H. Learning impairment in the radial-arm maze following prolonged cannabis treatment in rats. Psychopharmacology (Berl) 1982;77:117-23. [PubMed: 6289370]

Stiglick A, Kalant H. Behavioral effects of prolonged administration of delta 9-tetrahydrocannabinol in the rat. Psychopharmacology (Berl) 1983;80:325-30. [PubMed: 6312481]

Stiglick A, Kalant H. Residual effects of chronic cannabis treatment on behavior in mature rats. Psychopharmacology (Berl) 1985;85:436-9. [PubMed: 3927340]

Swift W, Coffey C, Carlin JB, Degenhardt L, Patton GC. Adolescent cannabis users at 24 years: Trajectories to regular weekly use and dependence in young adulthood. Addiction 2008;103:136170. [PubMed: 18855826]

Tapert SF, Schweinsburg AD, Drummond SP, Paulus MP, Brown SA, Yang TT, Frank LR. Functional MRI of inhibitory processing in abstinent adolescent marijuana users. Psychopharmacology (Berl) 2007;194:173-83. [PubMed: 17558500] 
Tarazi FI, Baldessarini RJ. Comparative postnatal development of dopamine $\mathrm{D}(1), \mathrm{D}(2)$ and $\mathrm{D}(4)$ receptors in rat forebrain. Int J Dev Neurosci 2000;18:29-37. [PubMed: 10708903]

Varvel SA, Cravatt BF, Engram AE, Lichtman AH. Fatty acid amide hydrolase (-/-) mice exhibit an increased sensitivity to the disruptive effects of anandamide or oleamide in a working memory water maze task. J Pharmacol Exp Ther 2006;317:251-7. [PubMed: 16352706]

Verrico CD, Jentsch JD, Roth RH, Taylor JR. Repeated, intermittent delta(9)-tetrahydrocannabinol administration to rats impairs acquisition and performance of a test of visuospatial divided attention. Neuropsychopharmacology 2004;29:522-9. [PubMed: 14694348]

Wiley JL, Buhler KG, Lavecchia KL, Johnson KM. Pharmacological challenge reveals long-term effects of perinatal phencyclidine on delayed spatial alternation in rats. Prog Neuropsychopharmacol Biol Psychiatry 2003;27:867-73. [PubMed: 12921921]

Wiley JL, Compton AD, Golden KM. Separation of drug effects on timing and behavioral inhibition by increased stimulus control. Experimental and Clinical Psychopharmacology 2000;8:451-61. [PubMed: 11127417]

Wiley JL, Willmore CB. Effects of nitric oxide synthase inhibitors on timing and short-term memory in rats. Behav Pharmacol 2000;11:421-9. [PubMed: 11103894]

Wittchen HU, Behrendt S, Hofler M, Perkonigg A, Lieb R, Buhringer G, Beesdo K. What are the high risk periods for incident substance use and transitions to abuse and dependence? Implications for early intervention and prevention. Int J Methods Psychiatr Res 2008;17:S16-29. [PubMed: 18543359] 

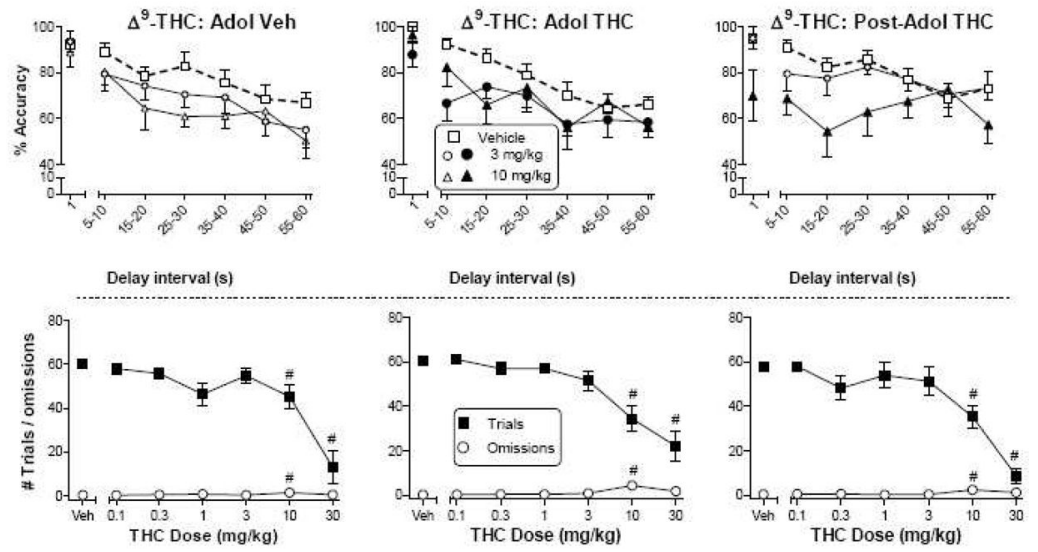

Figure 1.

Effects of $\Delta^{9}$-THC on accuracy (top panels) as a function of delay interval in a DNMP procedure in rats treated with vehicle (left panels) or $\Delta^{9}$-THC during adolescence (PN21-50, middle panels) or with $\Delta^{9}$-THC after adolescence (PN50-79, right panels). For each treatment group, numbers of trials and omissions are shown as a function of $\Delta^{9}$-THC dose (bottom panels). Values represent the mean ( \pm SEM) accuracy or number of trials or omissions. For all doses, $\mathrm{n}=10-11$ rats. In the top panels, the vehicle condition is shown by bold dashed line. Significant dose effects compared to vehicle are indicated by filled symbols. In bottom panels, \# indicates a significant main effect of dose for trial number and/or omissions $(\mathrm{p}<0.05$ compared to corresponding vehicle). 

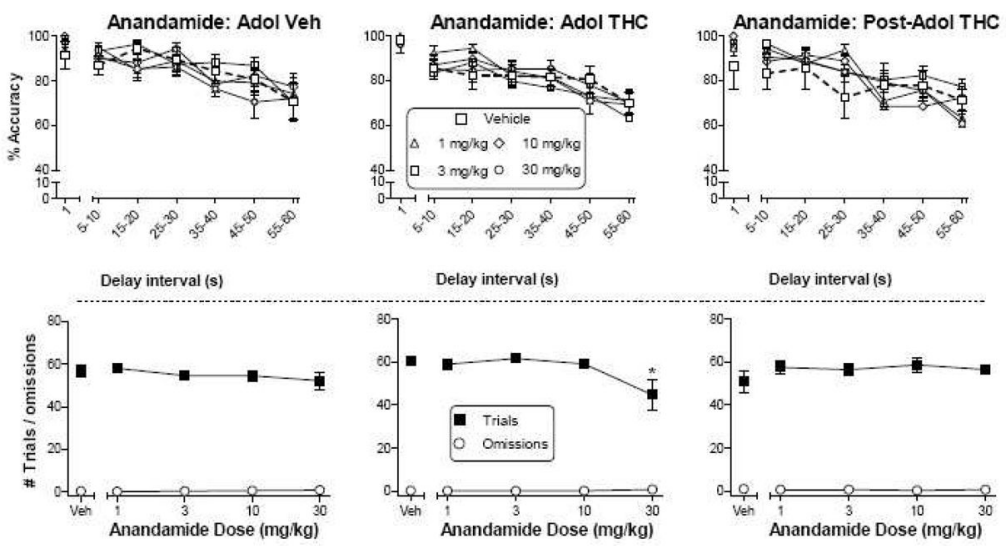

Figure 2.

Effects of anandamide on accuracy (top panels) as a function of delay interval in a DNMP procedure in rats treated with vehicle (left panels) or $\Delta^{9}$-THC during adolescence (PN21-50, middle panels) or with $\Delta^{9}$-THC after adolescence (PN50-79, right panels). For each treatment group, numbers of trials and omissions are shown as a function of anandamide dose (bottom panels). Values represent the mean $( \pm \mathrm{SEM})$ accuracy or number of trials or omissions. For all doses, $\mathrm{n}=10-11$ rats. In the top panels, the vehicle condition is shown by bold dashed line. Anandamide did not produce significant decreases in accuracy in any treatment group. In bottom panels, \# indicates a significant main effect of dose for trial number and/or omissions ( $<<0.05$ compared to corresponding vehicle). * indicates a group $\mathrm{X}$ dose interaction with significant difference of indicated dose from vehicle only in the rats treated with $\Delta^{9}$-THC during adolescence ( $\mathrm{p}<0.05$ compared with vehicle). 

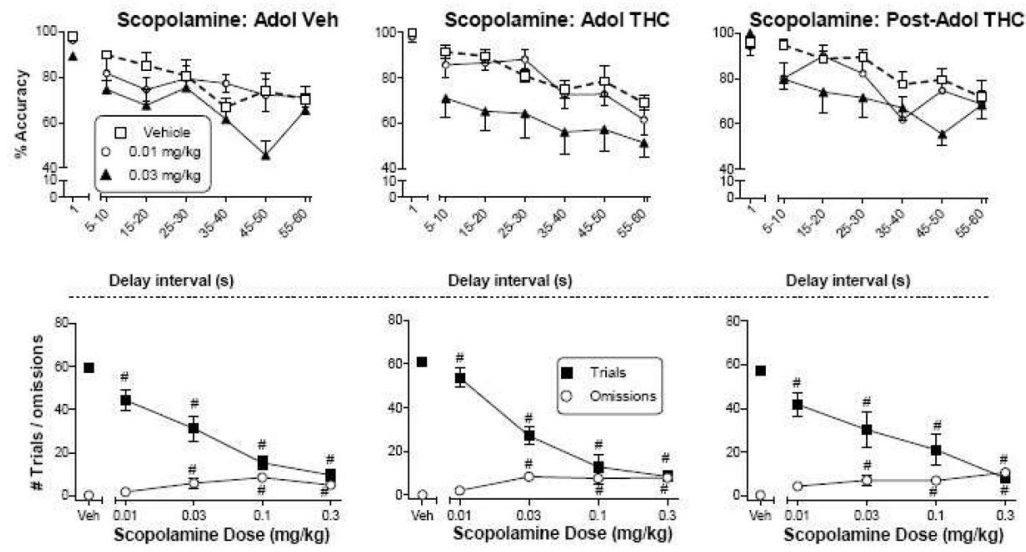

Figure 3.

Effects of scopolamine on accuracy (top panels) as a function of delay interval in a DNMP procedure in rats treated with vehicle (left panels) or $\Delta^{9}$-THC during adolescence (PN21-50, middle panels) or with $\Delta^{9}$-THC after adolescence (PN50-79, right panels). For each treatment group, numbers of trials and omissions are shown as a function of scopolamine dose (bottom panels). Values represent the mean ( \pm SEM) accuracy or number of trials or omissions. For all doses, $\mathrm{n}=10-11$ rats. In the top panels, the vehicle condition is shown by bold dashed line. In bottom panels, \# indicates a significant main effect of dose for trial number and/or omissions $(\mathrm{p}<0.05$ compared to corresponding vehicle). 

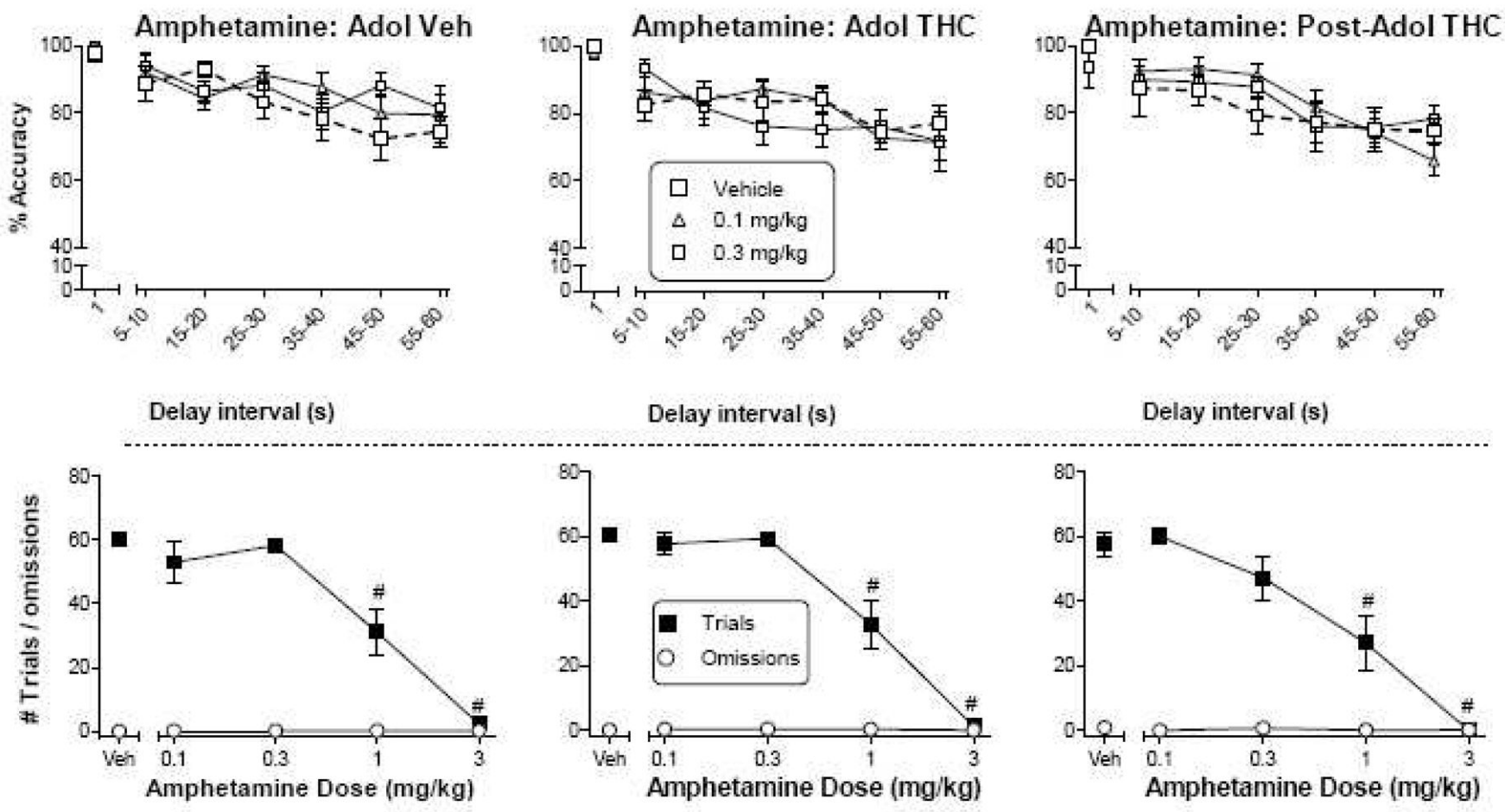

Figure 4.

Effects of amphetamine on accuracy (top panels) as a function of delay interval in a DNMP procedure in rats treated with vehicle (left panels) or $\Delta^{9}$-THC during adolescence (PN21-50, middle panels) or with $\Delta^{9}$-THC after adolescence (PN50-79, right panels). For each treatment group, numbers of trials and omissions are shown as a function of amphetamine dose (bottom panels). Values represent the mean $( \pm$ SEM) accuracy or number of trials or omissions. For all doses, $n=9-10$ rats. In the top panels, the vehicle condition is shown by bold dashed line.

Amphetamine did not produce significant decreases in accuracy in any treatment group. In bottom panels, \# indicates a significant main effect of dose for trial number and/or omissions ( $\mathrm{p}<0.05$ compared to corresponding vehicle). 